\title{
CONTROLE DO CRESCIMENTO VEGETATIVO E FLORAÇÃO DE MANGUEIRAS CV. KENT COM REGULADORES DE CRESCIMENTO VEGETAL ${ }^{1}$
}

\author{
MARIA APARECIDA DO CARMO MOUCO², ELIZABETH ORIKA ONO ${ }^{3}$, \\ JOÃO DOMINGOS RODRIGUES ${ }^{4}$
}

\begin{abstract}
RESUMO - O paclobutrazol é utilizado para o manejo da produção de manga na maioria dos pomares, com aplicação no solo, o que contribui para a maior persistência no ambiente. Assim, este trabalho teve como objetivo avaliar o efeito de reguladores de crescimento vegetal aplicado via foliar, prohexadione-Ca, etil-trinexapac e cloreto de chlormequat, e via solo, paclobutrazol, no controle da brotação vegetativa e na floração de mangueiras 'Kent'. O experimento foi conduzido na região do Vale do São Francisco, em Petrolina-PE, no período de dezembro de 2007 a julho de 2008, em mangueiras cultivadas no espaçamento de $8 \mathrm{~m}$ nas entrelinhas e $5 \mathrm{~m}$ entre plantas. Foram avaliados os efeitos dos tratamentos com reguladores vegetais aplicados via foliar e via solo, com relação ao vigor (número, comprimento e número de folhas dos novos fluxos vegetativos) e à floração (percentagem e comprimento das panículas). Verificou-se que o tratamento com paclobutrazol, combinado com o prohexadione-Ca, foi mais eficiente no controle do crescimento vegetativo dos ramos da mangueira 'Kent', mas não com relação à floração, em que não foram observadas diferenças siginificativas entre os tratamentos.
\end{abstract}

Termos para indexação: giberelina, prohexadione-Ca, etil-trinexapac, cloreto de chlormequat.

\section{CONTROL OF VEGETATIVE GROWTH AND FLOWERING ON MANGO CV. KENT WITH PLANT GROWTH REGULATORS}

\begin{abstract}
Paclobutrazol applied to the soil is used for the production management of mangoes in most of the orchards, but it presents as disadvantage the greater persistence in plant and soil. Three growth regulators (prohexadione-Ca, trinexapac-ethyl and chlormequat chloride) were foliar applied, at two dosages and compared to paclobutrazol applied as soil-drench. The experiment was conducted in the region of the São Francisco Valley, Petrolina, PE, from December 2007 to July 2008, in a mango orchard that has a spacing of $8 \mathrm{~m}$ between lines and $5 \mathrm{~m}$ between plants. In order to compare the effects of the treatment, data were evaluated regarding to vegetative growth (number, length and leaf number of vegetative branches) and flowering (percentage and panicles length). The combination of PBZ, soil applied with ProCa, foliar applied was the most efficient in controlling vegetative growth of branches of the mango 'Kent', but not regarding to flowering, where there were no differences between treatments.
\end{abstract}

Index terms: gibberellin, prohexadione-Ca, trinexapac-ethyl, chlormequat chloride.

\section{INTRODUÇÃO}

Os reguladores vegetais são substâncias químicas que têm sido utilizadas para controlar o crescimento vegetativo de algumas fruteiras de clima temperado (macieira, pereira), subtropical e tropical (abacateiro, citros e mangueira). Vale ressaltar que o manejo do crescimento vegetativo é de grande importância na produção de fruteiras, já que, evitando-se a brotação excessiva, podem-se promover a floração e a frutificação precoce em plantas jovens (DAVENPORT, 2007), como também reduzir o impacto negativo na qualidade e vida pós- colheita dos frutos e permitir bom desenvolvimento e porte da planta (GREENE, 2010). Na promoção à floração e produção de mangueiras sob condições de altas temperaturas, no semiárido nordestino, é indispensável o manejo em plantas com ramos maduros, no mínimo com três meses de idade (MOUCO et al., 2010a) .

Dos reguladores vegetais utilizados em frutíferas, o paclobutrazol (PBZ) apresenta o inconveniente de ser aplicado no solo, com a maior persistência deste produto, tanto na planta como no solo. Dos reguladores (retardantes) vegetais que interferem na síntese da giberelina 
(GA) podem ser relacionados: os compostos quaternários, como o cloreto de mepiquat e o cloreto de chlormequat (CCC), os compostos cíclicos contendo um nitrogênio, como o paclobutrazol (PBZ) e o uniconazole, e os acilciclohexanodionas, como o etil-trinexapac (TrixE) e o prohexadione-Ca (ProCa), que podem bloquear as reações finais do metabolismo de GA (ASIN et al., 2007).

Vale ressaltar que pesquisa com reguladores vegetais apresenta a rápida degradação do ProCa, o que torna necessário mais aplicações no controle do crescimento vegetativo em fruteiras ao longo do ciclo (GREENE, 2010). Asin et al. (2007) reportam que o efeito do ProCa foi mais rápido em reduzir a emissão e o comprimento dos ramos vegetativos da pereira em relação ao $\mathrm{PBZ}$, que foi mais persistente, sugerindo a combinação dos dois compostos como estratégia na redução do vigor desta cultura. Mouco et al. (2010b) testaram os reguladores de crescimento vegetal em mangueiras com seis anos de idade, observando que o ProCa e CCC aplicados via foliar anteciparam a floração e a colheita em 15 dias, enquanto no tratamento com PBZ aplicado no solo, a resposta foi em 25 dias, quando comparado com os tratamentos com TrixE e o controle. Este trabalho teve como objetivo avaliar o efeito de reguladores vegetais aplicados via foliar e no solo quanto à eficiência no controle da brotação vegetativa e na floração de mangueiras 'Kent'.

\section{MATERIAL E MÉTODOS}

O experimento foi conduzido em um pomar comercial de mangueiras cultivar Kent, no período de dezembro de 2007 a julho de 2008. A área localiza-se no município de Petrolina, situado na região do Vale do São Francisco, nas coordenadas geográficas a $09^{\circ}$ 24' de latitude Sul e $40^{\circ} 20^{\prime}$ de longitude a Oeste de Greenwich, com altitude de $370 \mathrm{~m}$. O clima da região é classificado, segundo Köeppen, como tipo Bswh, que corresponde à região semiárida muito quente. O índice pluviométrico anual é de 571,5 $\mathrm{mm}$, distribuído entre os meses de dezembro a abril. A temperatura média anual é de $26,4^{\circ} \mathrm{C}$, com média das mínimas de $20,6^{\circ} \mathrm{C}$ e média das máximas de $31,7^{\circ} \mathrm{C}$. Os solos são de textura arenosa, profundos, classificados como Neossolos Quartzarênicos.

$\mathrm{Na}$ época da instalação do experimento, as mangueiras conduzidas em espaçamento de $8 \mathrm{~m}$ entre linhas e $5 \mathrm{~m}$ entre plantas estavam com quatro anos de idade.

Foram avaliados quatro reguladores vegetais, sendo três deles aplicados em pulverizações foliares (duas doses): prohexadione-Ca (ProCa), etil- trinexapac (TrixE) e cloreto de chlormequat (CCC) e o paclobutrazol (PBZ) aplicado via solo (manejo do produtor). Também foram incluídos um tratamento com o PBZ aplicado via solo e uma pulverização com o ProCa e o controle. Os tratamentos estão descritos a seguir: T1- ProCa (1,5 g i.a. planta $\left.{ }^{-1}\right)$; T2- ProCa (3,0 g i.a. planta $\left.{ }^{-1}\right)$; T3- TrixE (1,0 g i.a. planta $\left.^{-1}\right)$; T4- TrixE (2,0 g i.a. planta-1 $)$; T5- CCC (1,5 g i.a. planta $\left.^{-1}\right)$; T6- CCC (3,0 g i.a. planta $\left.{ }^{-1}\right)$; T7- PBZ (4,0 g i.a. planta-1 $)$; T8- Controle; T9- PBZ (4,0 g i.a. planta $\left.^{-1}\right)+\operatorname{ProCa}\left(1,5\right.$ g i.a. planta $\left.^{-1}\right)$.

As aplicações dos reguladores vegetais, tanto via solo como foliar, foram realizadas a partir do primeiro fluxo vegetativo emitido após a poda de produção do pomar. Os tratamentos com o regulador vegetal ProCa foram aplicados em quatro pulverizações (3-12-2007; 21-12-2007; 11-012008; 31-01-2008), a intervalos de 20 dias. Foram realizadas duas aplicações de TrixE (03-12-2007; 17-01-2008), a intervalo de 45 dias. Os tratamentos com CCC foram aplicadas em três pulverizações (0312-2007; 03-01-2008; 1 1-02-2008), a intervalos de 30 dias. Já o PBZ foi aplicado via solo em única vez, em 03-12-2007, isolado e também associado com uma pulverização de ProCa, na dose de 1,5 g i.a. planta $^{-1}$.

O paclobutrazol (PBZ) foi utilizado na forma de Cultar, que tem $25 \%$ de i.a. O prohexadioneCa (ProCa) foi aplicado na forma de Viviful, contendo $27,5 \%$ de i.a. Já o Moddus 250 EC foi utilizado como fonte do etil trinexapac (TrixE) e o Tuval, como fonte de Cloreto de chlormequat (CCC).

O delineamento experimental foi o em blocos ao acaso, com nove tratamentos e quatro repetições, utilizando duas plantas por parcela. Foram identificados 16 ramos na parte mediana da copa, considerando quatro por quadrante para a avaliação dos tratamentos em resposta ao vigor (número de fluxos vegetativos, comprimento e número de folhas dos novos fluxos vegetativos) e a floração (percentagem e comprimento das panículas florais).

Durante a condução do experimento, as práticas culturais, como irrigação, adubação, tratos fitossanitários e manejo da floração, foram aquelas preconizadas para as condições semiáridas brasileiras. A lâmina de irrigação foi determinada estimando-se a evapotranspiração da cultura, considerando a eficiência do sistema de irrigação utilizado, como a vazão e o número de emissores por planta. Após 30 dias da aplicação do regulador vegetal, a lâmina diária foi reduzida em $50 \%$, sendo mantida até o florescimento das plantas (no mínimo $50 \%$ dos ramos com inflorescência), aos 230 dias após a aplicação dos tratamentos. Durante o período mencionado (da aplicação do regulador vegetal até a 
floração), foi aplicada uma lâmina total de 390 mm. A adubação foi feita baseada na análise de solo e foliar, com aplicação de $80 \mathrm{~g}$ de $\mathrm{P}_{2} \mathrm{O}_{5}, 30 \mathrm{~g}$ de $\mathrm{K}_{2} \mathrm{O}$ e $20 \mathrm{~L}$ de esterco, realizada após a colheita da safra anterior e da poda de produção (40 dias antes da implantação do experimento).

Para comparação das médias das variáveis estudadas, utilizou-se o teste de Tukey, ao nível de $5 \%$ de probabilidade, utilizando-se do programa 'Statistica for Windows' versão 5.5 (Statsoft).

\section{RESULTADOS E DISCUSSÃO}

Nas avaliações da brotação vegetativa iniciadas aos 15 dias após a aplicação dos reguladores vegetais, pôde-se observar que todas as plantas dos tratamentos apresentavam pelo menos um novo fluxo vegetativo; assim, a partir deste resultado, pode-se verificar a importância do manejo da mangueira após a emissão de vários fluxos vegetativos e não apenas de um fluxo, como conduzido neste trabalho. De acordo com Bower et al. (1990), em fruteiras tropicais e subtropicais, como abacateiro, citros e mangueira, os hormônios vegetais e o balanço de carboidratos endógenos são responsáveis pelo crescimento vegetativo, floração e fixação de frutos.

Em mangueira, Davenport (2007) reporta que, após a colheita, ocorre brotação natural das gemas; nesta fase, os níveis de carboidrato nos ramos são reduzidos, assim como o amido, que permanece baixo até a emissão da gema floral.

Verificou-se que a temperatura média do início do experimento até a primeira indução (aplicação do indutor à brotação, depois do repouso dos ramos), entre 3 de dezembro de 2007 e 23 de abril de 2008 , variou de 30 a $35^{\circ} \mathrm{C}$ (dia) e 22 a $25^{\circ} \mathrm{C}$ (noite), condição favorável à emissão de brotações vegetativas na mangueira (DAVENPORT, 2007).

Os tratamentos com a aplicação de PBZ isolado e associado com o ProCa reduziram significativamente o comprimento do fluxo vegetativo, em $39,5 \%$ e $67,5 \%$, respectivamente, em relação ao controle (Tabela 1). O ProCa, considerado instável e com curto tempo de persistência na planta, em torno de 10 a 14 dias em macieiras, induz resposta rápida na inibição do crescimento vegetativo (ZADRAVEC et al., 2008; GREENE, 2008, 2010), e principalmente quando associado ao PBZ, aplicado via solo, permite maior eficiência no controle do vigor das brotações.

No segundo fluxo vegetativo, emitido após as pulverizações dos tratamentos, observou-se que o ProCa não alterou significativamente o comprimento dos ramos em relação ao controle, enquanto as plantas tratadas com TrixE emitiram segundo fluxo vegetati- vo mais longo, possivelmente pelo tempo transcorrido depois da segunda e última pulverização, aos 70 dias. Fagerness e Yelverton (2000) reportam também que uma aplicação de TrixE inibe temporariamente o crescimento de Gramíneas, mas o efeito desaparece em quatro semanas após a primeira aplicação, e que existe maior taxa de crescimento após o período de inibição, podendo anular as diferenças no final do período avaliado.

Pode-se verificar que aplicação com o cloreto de chlormequat (CCC), na dose de 1,5 g i.a. planta $^{-1}$ reduziu o comprimento do segundo ramo em relação ao primeiro emitido logo após a primeira pulverização, em 50\%. Observou-se ainda a redução do tamanho da brotação em $38,78 \%$ em relação aos ramos das plantas do controle (Tabela 1). Maas (2006) reporta que em pereiras, aplicações de CCC, em anos consecutivos, reduzem progressivamente o tamanho e o número de fluxos vegetativos emitidos pela planta. Já na dose de $3,0 \mathrm{~g}$ i.a. planta $^{-1}$, houve efeito inverso na regulação do crescimento vegetativo. Estes autores recomendam a realização de novos trabalhos com a utilização de CCC em dose igual ou inferior a $1,5 \mathrm{~g}$ i.a. planta $^{-1}$.

Os tratamentos com reguladores vegetais incrementaram o número de folhas do fluxo emitido pelas plantas em relação ao controle, contudo sem diferir significativamente. Tal resultado foi observado por Ramirez et al.(2006) utilizando o ProCa em macieiras. Vale ressaltar que o número de folhas presentes em cada fluxo vegetativo em mangueira pode variar entre cultivares, como também é influência da temperatura. Mangueiras cultivadas sob temperaturas de $20^{\circ} \mathrm{C} / 15^{\circ} \mathrm{C}$ produzem, em média, 7,1 folhas por fluxo vegetativo, enquanto em condições de $30^{\circ} \mathrm{C} / 25^{\circ} \mathrm{C}$, a média é de 13,6 folhas por fluxo (WHILEY, 1993). Neste trabalho, a média foi 12 folhas por fluxo vegetativo (Tabela 1).

A floração irregular das plantas do experimento comprometeu a análise da percentagem de ramos com inflorescências (panículas). No entanto, nas avaliações de ocorrência de brotações, aos 230 dias após a aplicação dos tratamentos, pôde-se observar tendência da dose de 3,0 g i.a. planta $^{-1}$ com os reguladores vegetais ProCa e CCC em incrementar a floração, quando comparado com a dose de 1,5 g i.a. planta $^{-1}$ (Figura 1). Já as plantas tratadas com dose de 2,0 g i.a. planta $^{-1}$ de TrixE não apresentaram novas brotações vegetativas ou florais, até o final das avaliações, podendo-se relacionar aos danos causadas nas gemas, observadas em campo, sugerindo evitar dose mais alta com este regulador vegetal. Pôde-se observar que plantas tratadas com o PBZ, quando combinado com o ProCa, manteve mais ramos em 
repouso (não vegetaram) que as tratadas somente com o PBZ.

Neste experimento, analisou-se apenas o dado referente ao comprimento das inflorescências (Tabela 1), em que somente o TrixE, na dose de 2,0 g i.a. planta $^{-1}$, reduziu significativamente o tamanho das panículas em relação ao controle. Verificou-se ainda que as plantas deste tratamento apresentaram poucas inflorescências (Figura 1), além da queima das bordas das folhas e das gemas, compactação das inflorescências $(64,4 \%)$, quando comparado ao tratamento com dose de 1,0 g i.a. planta $^{-1}$ (Tabela 1).

De acordo com Mouco e Albuquerque (2005), altas doses de PBZ utilizadas na cultura da mangueira, principalmente no manejo visando à produção no primeiro semestre do ano, tendem a compactar as panículas. No entanto, o ProCa combinado com o PBZ minimiza este efeito quando comparado com o tratamento com PBZ isolado.

TABELA 1 - Média de comprimento do primeiro e segundo fluxo vegetativo (cm), número de folhas e comprimento médio das panículas de mangueiras 'Kent', após a aplicação dos tratamentos com reguladores de crescimento vegetal. Fazenda Andorinhas, Petrolina-PE. 2008

\begin{tabular}{|c|c|c|c|c|}
\hline Tratamentos & $\begin{array}{c}1^{\circ} \text { fluxo } \\
\text { vegetativow } \\
(\mathrm{cm})\end{array}$ & $\begin{array}{c}2^{\circ} \text { fluxo } \\
\text { vegetativo }^{\mathrm{y}}(\mathrm{cm})\end{array}$ & $\begin{array}{l}\text { Folhas } \\
\mathbf{1}^{\circ} \text { fluxo }\end{array}$ & $\begin{array}{c}\text { Panícula } \\
(\mathrm{cm})\end{array}$ \\
\hline 1-ProCa1 (1,5g i.a. planta $\left.^{-1}\right)$ & $16,55 \mathrm{a}^{\mathrm{z}}$ & $17,75 \mathrm{ab}$ & $16,69 \mathrm{a}$ & $30,29 \mathrm{ab}$ \\
\hline 2-ProCa2 $\left(3,0\right.$ g i.a. planta $\left.^{-1}\right)$ & $17,54 \mathrm{a}$ & $11,29 \mathrm{ab}$ & $14,50 \mathrm{a}$ & $41,24 \mathrm{a}$ \\
\hline 3-TrixE1 (1,0 g i.a. planta $\left.{ }^{-1}\right)$ & $14,17 \mathrm{ab}$ & $18,30 \mathrm{a}$ & $14,31 \mathrm{a}$ & $37,26 \mathrm{a}$ \\
\hline 4-TrixE2 $\left(2,0\right.$ g i.a. planta $\left.^{-1}\right)$ & $14,73 \mathrm{a}$ & $18,07 \mathrm{a}$ & $14,81 \mathrm{a}$ & $13,25 \mathrm{~b}$ \\
\hline 5-CCC1 $\left(1,5\right.$ g i.a. planta $\left.{ }^{-1}\right)$ & $16,49 \mathrm{a}$ & $8,62 \mathrm{~b}$ & $15,37 \mathrm{a}$ & $28,54 \mathrm{ab}$ \\
\hline 6-CCC2 $\left(3,0\right.$ g i.a. planta $\left.^{-1}\right)$ & $16,31 \mathrm{a}$ & $14,90 \mathrm{ab}$ & $15,69 \mathrm{a}$ & $29,49 \mathrm{ab}$ \\
\hline 7-PBZ (4,0 g i.a. planta-1) & $10,24 \mathrm{~b}$ & $15,80 a b$ & $15,87 \mathrm{a}$ & $29,75 \mathrm{ab}$ \\
\hline 8-Controle & $16,94 \mathrm{a}$ & $14,08 \mathrm{ab}$ & $13,75 \mathrm{a}$ & 39,64 a \\
\hline 9-PBZ + ProCa1 & $5,50 \mathrm{c}$ & $12,56 \mathrm{ab}$ & $13,83 \mathrm{a}$ & $37,93 \mathrm{a}$ \\
\hline C V (\%) & 12,4 & 22,4 & 12,3 & 20 \\
\hline
\end{tabular}

w Primeira fluxo vegetativo após a aplicação dos tratamentos.

${ }^{y}$ Segunda brotação vegetativa após a aplicação dos tratamentos.

${ }^{z}$ Média de quatro repetições de 16 ramos.planta ${ }^{-1}$. Médias seguidas pela mesma letra nas colunas não são estatisticamente diferentes (Tukey $P \leq 0,05$ ).

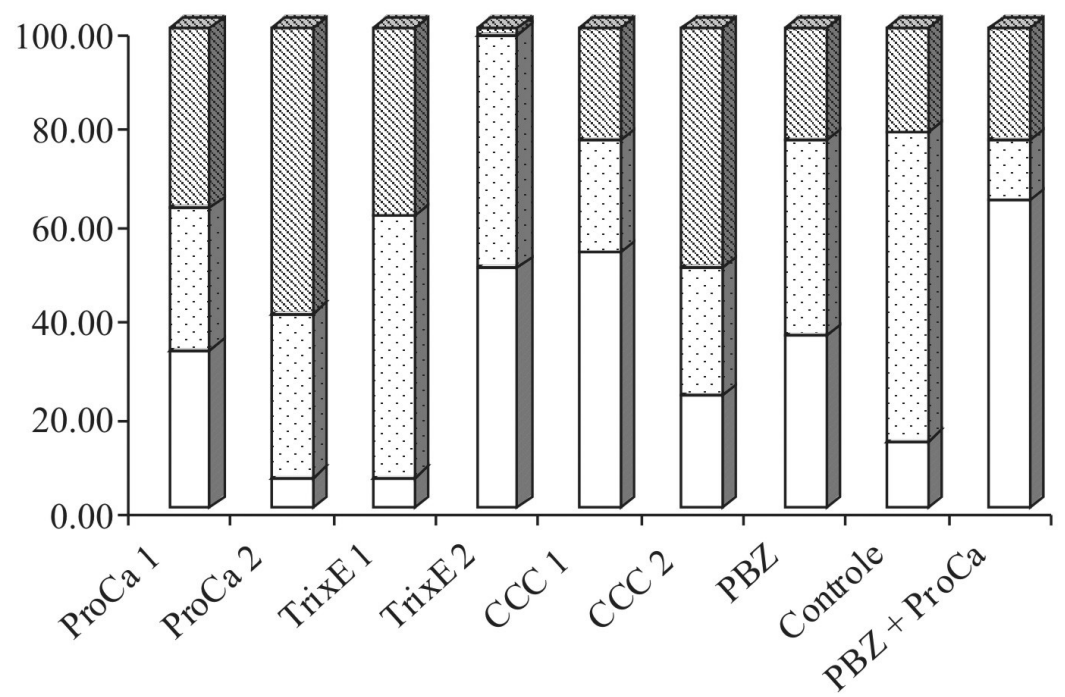

$\square$ Repouso $\square$ Brotação vegetativa $\quad$ Floração

FIGURA 1 - Relação entre a ocorrência de ramos em repouso vegetativo, ramos com fluxos vegetativos e ramos com inflorescência, aos 230 dias do início do experimento, na segunda safra de mangueira cv. Kent. Fazenda Andorinhas. Petrolina-PE. 2008. 


\section{CONCLUSÃO}

O PBZ aplicado via solo, associado com uma pulverização de ProCa, na dose de 1,5g i.a. planta $^{-1}$, é mais eficiente no controle do crescimento vegetativo em ramos de mangueiras 'Kent'.

\section{REFERÊNCIAS}

ASIN, L.; ALEGRE, S.; MONTSERRAT, R. Effect of paclobutrazol, prohexadione-Ca, deficit irrigation, summer pruning and root pruning on shoot growth, yield, and return bloom, in a 'Blanquilla' pear orchard. Scientia Horticulturae, Amsterdam, v.113, n.2, p.142-148, 2007.

BOWER, J.P.; LOVATT, C.J.; CUTTING, J.G.M.; BLAKE, M.M. Interaction of plant growth regulator and carbohydrate in flowering and fruit set. Acta Horticulturae, The Hague, n.275, p.425-434, 1990.

DAVENPORT, T. L. Reproductive physiology of mango. Brazilian Journal of Plant Physiology, Piracicaba, v.19, n.4, 2007. Disponível em: $<$ http://www.scielo.br/scielo.php? script $=$ sci arttext\&pid=S1677-04202007000400007\&lng=es\&

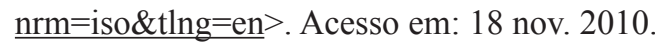

FAGERNESS, M.J.; YELVERTON, F.H. Tissue production and quality of 'Tifway' Bermudagrass as affected by seasonal application patterns of Trinexapac-Ethyl. Crop Science, Madison, v.40, p.493-497, 2000.

GREENE, D. W. The development and use of plant biorregulators in tree fruit production. Acta Horticulturae, The Hague, n.884, p.31-40, 2010.
GREENE, D.W. The effect ofrepeat annual applications of Prohexadione-calcium on Fruit Set, Return Bloo, and Fruit Size of Apples. Hortscience, Alexandria, v.43, p.286-583, 2008.

MAAS, F. Carry-over effects of CCC - applications in pear orchards. Acta Horticulturae, The Hague, n.727, p. 125-132, 2006.

MOUCO, M. A. do C.; ALBUQuerQue, J. A. S. de. Efeito do paclobutrazol em duas épocas de produção da mangueira. Bragantia, Campinas, v.64, n.2, p.219-225, 2005.

MOUCO, M.A do C.; ONO. E.O.; RODRIGUES, J.D. Inibidores de síntese de giberelinas e crescimento de mudas de mangueira 'Tommy Atkins'. Ciencia Rural, Santa Maria, v.40, p.273-279, 2010a.

MOUCO, M.A do C.; ONO. E.O.; RODRIGUES, J.D. Mango Flower Induction in the Brazilian Northeast Semi-Arid with Gibberellin Synthesis Inhibitors. Acta Horticulturae, The Hague, n.884, p.591-596, 2010b.

RAMÍREZ, H.; ALONSO, S.; BENAVIDES, A. Prohexadione-Ca modifies Growth and Endogenous Hormones in the shoot Apex in Apple Trees. Acta Horticulturae, The Hague, n. 727, p.117-123, 2006.

ZADRAVEC, P.; CMELIK, Z.; TOJNKO, S.; UNUK, T.; SCHLAUER, B. Vegetative growth, yield and fruit quality of 'Gala' apple treated with Regalis (prohexadione-Ca). Acta Horticulturae, The Hague, n.774, p.287-290, 2008.

WHILEY, A.W. Environmental effects on phenology and physiology of mango. A review. Acta Horticulturae, The Hague, n. 341, p.168-176, 1993. 